\title{
Corrosion Resistance of Silane-Modified Hydroxide Zinc Carbonate Film on AZ31 Magnesium Alloy
}

\author{
Rong-Chang Zeng • Li-Jun Liu • Ting-Ting Pang • Fen Zhang • Wei-Wei Zhang • Shuo-Qi Li • \\ Hong-Zhi Cui • En-Hou Han
}

Received: 12 July 2014/Revised: 20 November 2014/Published online: 13 January 2015

(C) The Chinese Society for Metals and Springer-Verlag Berlin Heidelberg 2015

\begin{abstract}
The corrosion resistance of magnesium alloys can be improved using functional surface modification such as hydrophobic treatment. In this study, a hierarchical hydroxide zinc carbonate (HZC) film was fabricated on AZ31 magnesium alloy via a simple chemical-bath deposition process using urea aqueous solution. The morphologies, compositions and corrosion resistance of the hydrophobic film were analyzed using scanning electron microscopy, X-ray diffraction and Fourier transform infrared spectrometer, and electrochemical measurements as well. The results revealed that the HZC film displayed flower-like protrusions and had a thickness of approximately $100 \mu \mathrm{m}$. The fluoroalkylsilane (FAS)-modified HZC film exhibited a hydrophobic property with a water contact angle of $131.3^{\circ}$. The FAS/HZC film significantly improved the corrosion resistance of the AZ31 alloy due to hierarchical structures and hydrophobic modification.
\end{abstract}

KEY WORDS: Magnesium alloy; Hydrophobicity; Silane; Surface modification; Corrosion

\section{Introduction}

Magnesium alloys have been utilized in automobile, aircraft and electronics industries due to their low density,

Available online at http://link.springer.com/journal/40195

R.-C. Zeng $(\bowtie) \cdot$ L.-J. Liu $\cdot$ T.-T. Pang $\cdot$ F. Zhang $\cdot$

W.-W. Zhang $\cdot$ S.-Q. Li $(\bowtie) \cdot$ H.-Z. Cui

College of Materials Science and Engineering, Shandong

University of Science and Technology, Qingdao 266590, China

e-mail: rczeng@foxmail.com

S.-Q. Li

e-mail: lishuoqi@sdust.edu.cn

R.-C. Zeng - F. Zhang $\cdot$ S.-Q. Li

State Key Laboratory of Mining Disaster Prevention and Control Co-founded by Shandong Province and the Ministry of Science and Technology, Shandong University of Science and

Technology, Qingdao 266590, China

E.-H. Han

Institute of Metals Research, Chinese Academy of Sciences,

Shenyang 110016, China high specific strength and damping characteristic [1-7]. However, the high chemical activity in aggressive environments is one of the major obstacles for the wide use of magnesium alloys [8-10]. Accordingly, the contact of the magnesium alloys with water triggers the corrosion reaction [11]. Thus, a hydrophobic coating is an effective approach to improve corrosion resistance of metals because it inhibits the contact of the metal surface with water and environment humidity. And thus, the hydrophobic coating may be applied on magnesium alloys $[12,13]$.

Hydrophobic surfaces have been attracted considerable interests due to its great importance in fundamental researches and industrial applications [14]. In the past decade, many researchers have focused on the influence of hydrophobic surfaces on self-cleaning, anti-icing and corrosion protection of metals [15-17]. The corrosion resistant mechanism of the hydrophobic surface is ascribed to the nanostructure on the hydrophobic surface easily trapping a large amount of air within the 'valleys' of the nanostructure. These 'air valleys' then prevent the migration of aggressive ions [18]. Based on this consideration, many methods have been proposed to prepare hydrophobic films 
on $\mathrm{Al}, \mathrm{Cu}$, steel and $\mathrm{Mg}$ substrates for an improvement in corrosion resistance [19].

Generally, the hydrophobicity of a surface can be achieved by both surface roughing and lowering surface energy [20, 21]. Various methods such as chemical etching [22], electro-spinning [23], chemical vapor deposition (CVD) [24], sol-gel process [25, 26] and layer-by-layer assembly [27, 28] have been used to prepare hierarchical hydrophobic surfaces on $\mathrm{Mg}$ alloys. However, some approaches may pollute the environment because of the use of strong acid [12] and cerium nitrate [29]. In addition, some reagents used are too expensive, and the process is too complex [30, 31]. Therefore, a highly low cost, environmentally friendly, safe and nontoxic method is needed.

In particular, chemical conversion coatings have outstanding advantages such as lower cost and simplicity in operation [32]. And according to our recent publications, phosphate conversion coatings are environmentally friendly and have been successfully exploited to protect magnesium alloys from corrosion [33-35]. However, the phosphate conversion coatings lack hydroxyl groups that allow further modification of functional molecules. Herein, we report a simple method for fabricating a hydroxide zinc carbonate $(\mathrm{HZC})\left(\mathrm{Zn}_{5}(\mathrm{OH})_{6}\left(\mathrm{CO}_{3}\right)_{2}\right)$ film with a hierarchical structure on magnesium alloy AZ31. The hydroxyl groups on the HZC surface make it easier for fluoroalkylsilane (FAS) modification. The hydrophobic properties of the hybrid FAS/HZC coating are improved due to the synergistic action of the rough morphology and the hydrophobic groups, and the corrosion resistance of magnesium alloys was improved finally.

\section{Experimental}

\subsection{Preparation of the HZC Film}

All chemicals were analytical grade. The as-extruded AZ31 magnesium alloy used has nominal chemical compositions: (2.5-3.0) wt $\% \mathrm{Al},(0.7-1.3) \mathrm{wt} \% \mathrm{Zn}, \geq 0.20 \mathrm{wt} \% \mathrm{Mn}$ and balanced $\mathrm{Mg}$. The samples with a dimension of $20 \mathrm{~mm} \times 20 \mathrm{~mm} \times 3 \mathrm{~mm}$ were polished with SiC papers up to 1,500 grit, washed with distilled water and ethanol, and dried in warm air. The HZC films were fabricated through a chemical-bath deposition process, which is reported by Liang et al. [36]. Briefly, the AZ31 substrate was immersed perpendicularly in $0.5 \mathrm{~mol} / \mathrm{L}$ zinc nitrate aqueous solution containing $0.25 \mathrm{~mol} / \mathrm{L}$ urea in a 3-neck, round-bottom flask, equipped with a Teflon-coated magnetic stir bar and reflux condenser, and stirred at $95{ }^{\circ} \mathrm{C}$ for $1.5 \mathrm{~h}$. Then, the prepared HZC-coated AZ31 substrate was washed with distilled water, dried by warm air, finally placed in an oven at $80{ }^{\circ} \mathrm{C}$ for $1 \mathrm{~h}$.

\subsection{Modification of the HZC Surfaces}

The chemical fluoroalkylsilane $\left[\mathrm{FAS}, \mathrm{CF}_{3}\left(\mathrm{CF}_{2}\right)_{7} \mathrm{CH}_{2}\right.$ $\mathrm{CH}_{2} \mathrm{Si}\left(\mathrm{OCH}_{3}\right)_{3}$ ] was hydrolyzed [37] prior to the coating process on the HZC-coated AZ31 substrates. A beaker containing a mixture of $100 \mathrm{~mL}$ distilled water and $1 \mathrm{~mL}$ FAS was placed in an ultrasonic bath for $30 \mathrm{~min}$. Hydrophobicity treatment was then carried out by immersing the HZC-coated AZ31 samples into the prepared FAS solutions in a Teflon-lined autoclave, which was then heated at $120{ }^{\circ} \mathrm{C}$ for $12 \mathrm{~h}$. After that, the substrates were rinsed with ethanol several times and then placed in the oven at $120{ }^{\circ} \mathrm{C}$ for $2 \mathrm{~h}$. The silane-modified HZC film was denoted as FAS/ HZC.

\subsection{Surface Characterization and Electrochemical Measurement}

For surface morphological characterization of the substrates, top and cross-sectional views were made through a field-emission scanning electron microscope (FE-SEM, Nova NanoSEM 450). Meanwhile, the affiliated energydispersive spectrometer (EDS) was used to analyze the elements on the specimens. The microstructures of the coated samples were analyzed by means of X-ray diffraction (XRD, Model D/Max 2500PC Rigaku), with a $\mathrm{Cu}$ target at a scanning rate of $8 \% \mathrm{~min}$. The diffraction patterns were obtained between $5^{\circ}$ and $80^{\circ}$. The chemical bonding of the surfaces was characterized through Fourier transform infrared spectroscopy (FT-IR, Nicolet 380, Thermo Electron Corporation). The water contact angle (CA) was measured using a contact angle goniometer (OCA15EC, Dataphysics Instruments $\mathrm{GmbH}$ ). The volume of one water drop was $2 \mu \mathrm{L}$. Electrochemical tests were performed on an electrochemical potentiostat (PAR Model 2273, Princeton) in a three-electrode system with the samples as the working electrode, a saturated calomel electrode (SCE) as the reference electrode and a platinum sheet as the counter electrode. The experiments were carried out in $3.5 \mathrm{wt} \% \mathrm{NaCl}$ solution at room temperature. The polarization curves were recorded with a scan rate of $2 \mathrm{mV} / \mathrm{s}$.

\section{Result and Discussion}

\subsection{Morphologies of Hydrophobic Magnesium Surfaces}

SEM micrographs of the coated samples are shown in Fig. 1. The HZC film has a considerable number of voids and flower-like protrusions with a diameter approximately of $10 \mu \mathrm{m}$ (Fig. 1a). The high magnification of a single flower (Fig. 1b) reveals that nanostructured plates are 

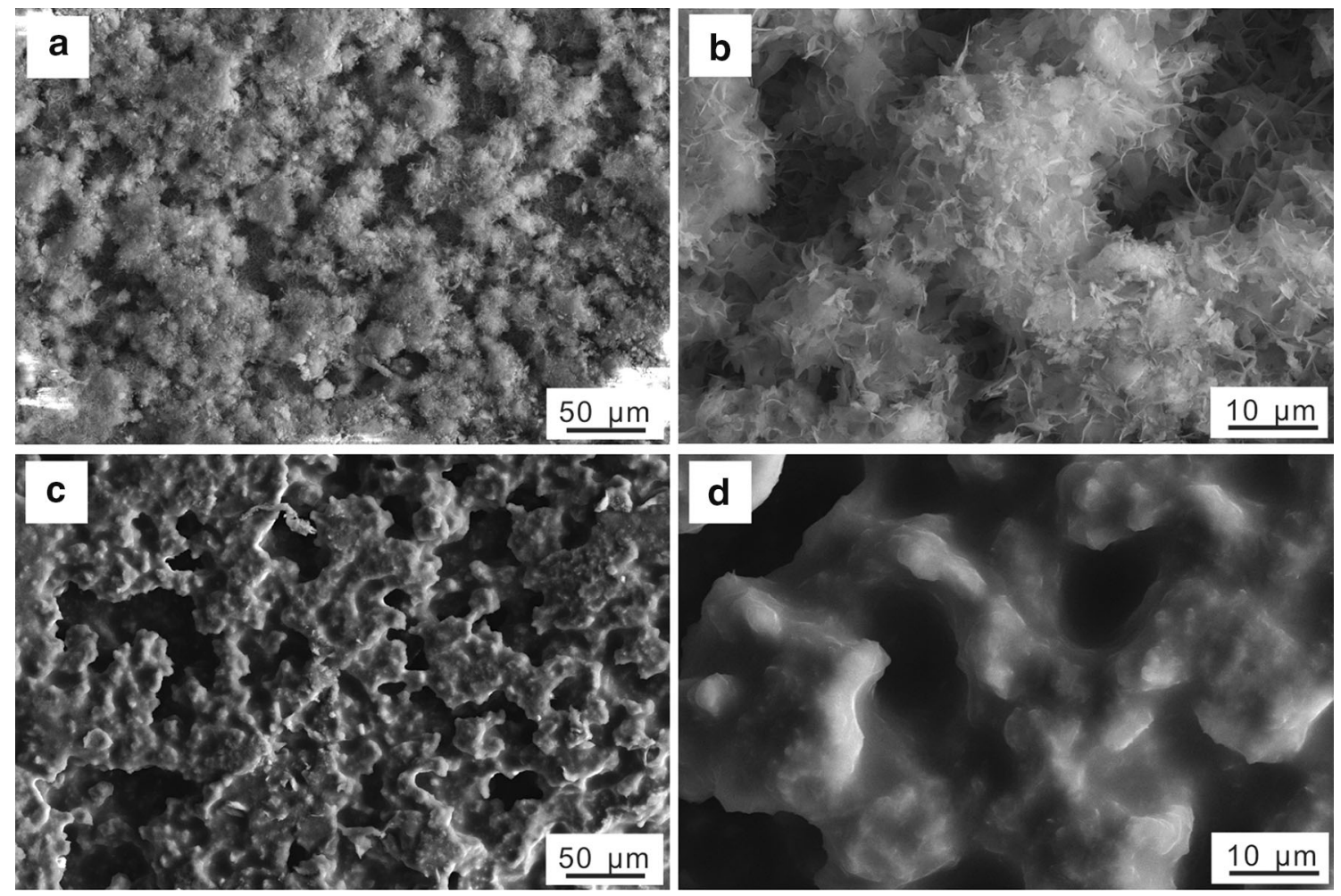

Fig. 1 SEM images of the HZC a, b and FAS/HZC films $\mathbf{c}, \mathbf{d}$

randomly dispersed. The hierarchical nanostructures may exhibit an apparent hydrophobicity when the surface was treated with FAS [38]. Figure 1c designates the SEM image of the FAS/HZC film. The silane-treated film still possessed a binary hierarchical nanostructure similar to lotus leaves (Fig. 1d).

The elemental mapping images and spot spectra disclose the elemental distribution of $\mathrm{C}, \mathrm{O}, \mathrm{Zn}$ and $\mathrm{Si}$ on the surface of the HZC and FAS/HZC films. As shown in Fig. 2a, the $\mathrm{Zn}$ element was uniformly distributed on the surface of the HZC-coated samples. The presence of $\mathrm{Zn}$ and $\mathrm{O}$ elements implies the HZC coatings successfully deposited on the substrate surface. The elemental mapping of the FAS/HZC film reveals the presence of uniformly distributed Si element (Fig. 2b), indicating the formation of a dense and uniform FAS film. It is noted that no cracks or delamination were observed on the FAS/HZC surface.

The microstructures of the coatings were further characterized by the cross-sectional profile using SEM and EDS analysis. As can be seen from Fig. 3a, the HZC coating with a thickness of about $100 \mu \mathrm{m}$ contains a number of pores and cracks. From the EDS line scanning across the layer and into the substrate, a uniform distribution of $\mathrm{Zn}$ and $\mathrm{O}$ elements through the deposited layer is readily observed. The FAS/HZC coating (Fig. 3b) seems more compact, which can be ascribed to the fact that fully cross- linked $\mathrm{Si}-\mathrm{O}-\mathrm{Si}$ layer formed in the voids of the HZC coatings. The corresponding distribution of $\mathrm{Si}$ element along the cross section confirmed that the FAS coatings were formed on both the surface and penetrated inside the voids of the HZC film. Namely, the Zn-rich HZC interlayer existed between the substrate and the FAS coatings. The total thickness of the Zn-rich interlayer and the FAS coatings is approximately $120 \mu \mathrm{m}$. The EDS analysis demonstrates that the FAS modification resulted in a substantial amount of silane permeated through the relatively porous HZC layer with massive hydroxyl groups on the surface, which may be helpful for an improvement in the corrosion resistance of the AZ31 substrate.

\subsection{Wettability}

The water CA value on a substrate is a good indicator of the wettability of a substrate [12]. The CA values of the surfaces display the change in the wettability of the AZ31 surfaces. As shown in Fig. 4a, the AZ31 substrate surface was hydrophilic with a CA of $60.5^{\circ}$. Interestingly, the CA of the HZC film rose up to $97.3^{\circ}$ (Fig. 4b). This result is attributed to the walls of the HZC nanostructured plates, mainly composed of zinc atoms, showing a relative hydrophobicity [39]. When the HZC film was modified with FAS, the CA had further increase and reached up to $131.3^{\circ}$ (Fig. 4c), while Xu et al. [18] reported a maximum 


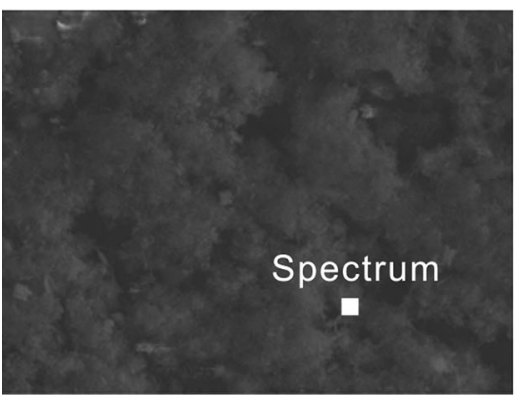

$90 \mu \mathrm{m}$
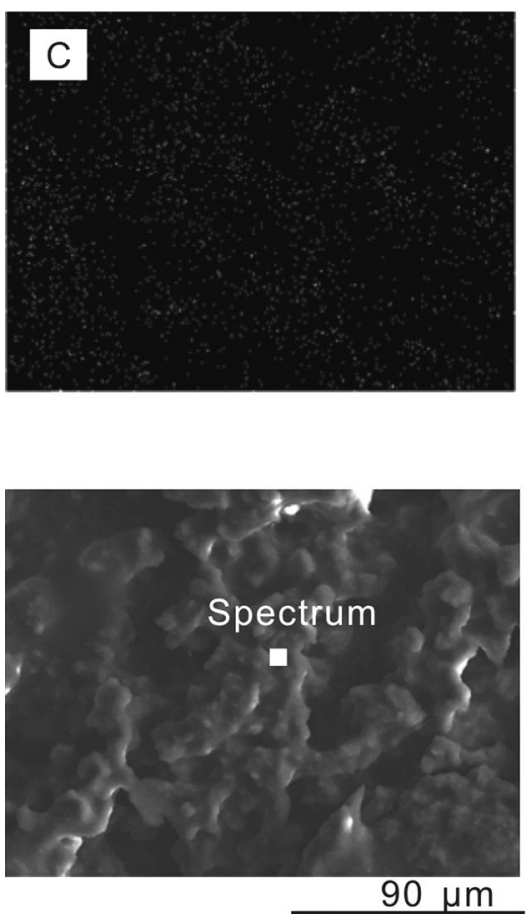

$90 \mu \mathrm{m}$

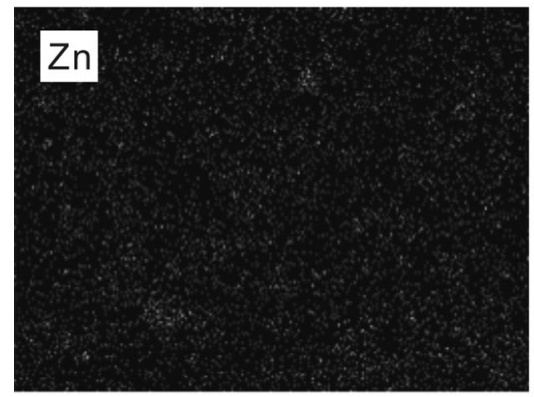

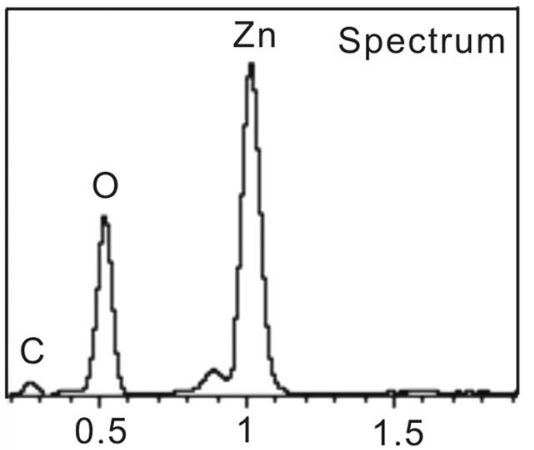
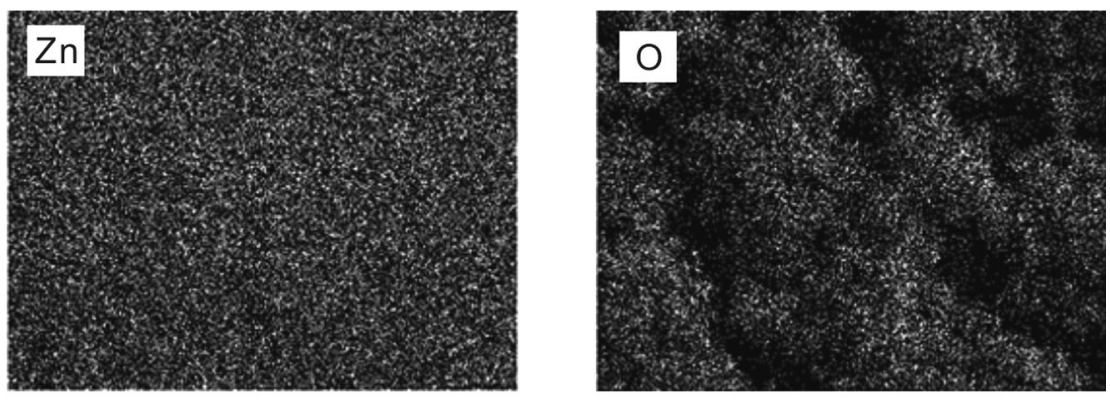

a
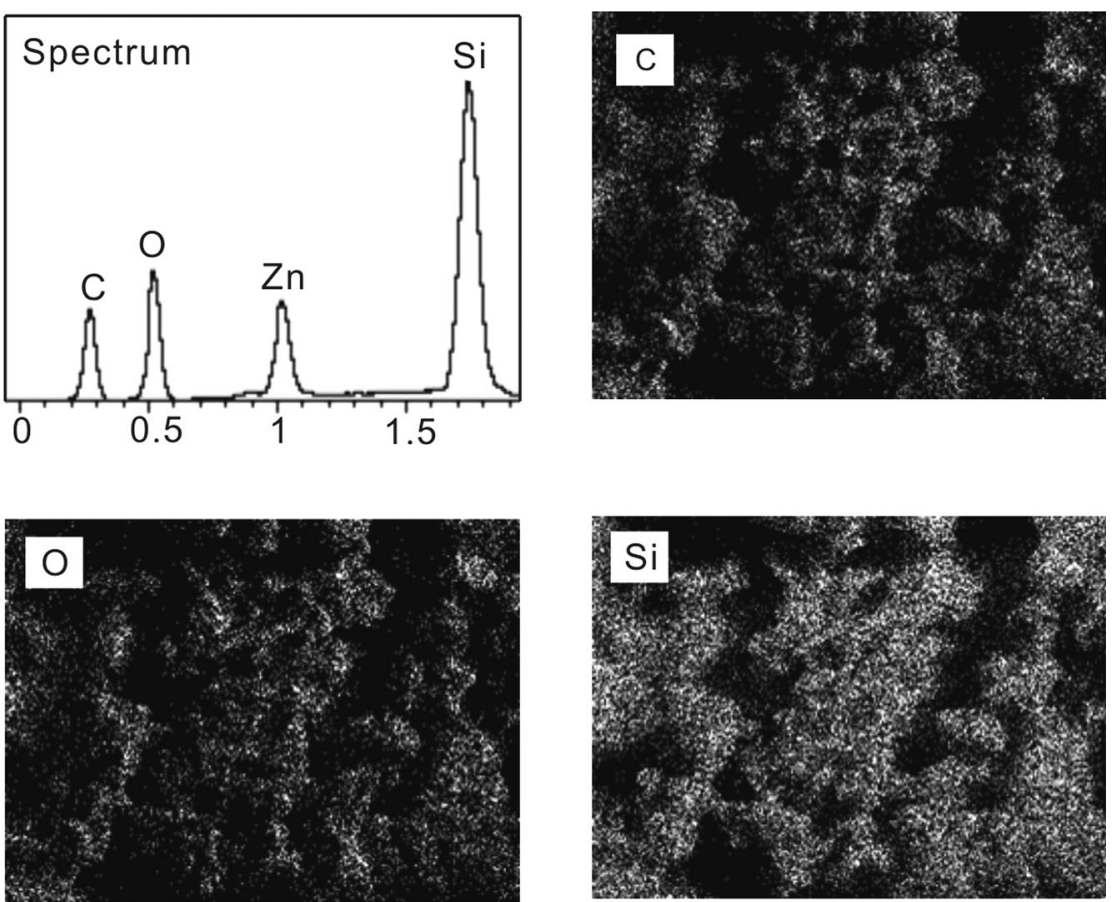

Fig. 2 Elemental mapping images and EDS spectra for the HZC film a and the FAS/HZC film b

CA of $165.2^{\circ}$ of a FAS film on the magnesium alloy surface experienced electrochemical machining. Despite a $19.7 \%$ decrease in the CA, the FAS-modified HZC coating attained a hydrophobic property, which was attributed to the combination of nanostructured HZC film and the low surface energy of the FAS film [40].

\subsection{Chemical Composition of the Hydrophobic Surface}

The chemical structures of the HZC film were characterized by virtue of XRD (Fig. 5). Compared with the AZ31 substrate (pattern a), the HZC film (pattern b) shows characteristic peaks of the HZC compound [41]. It is well 

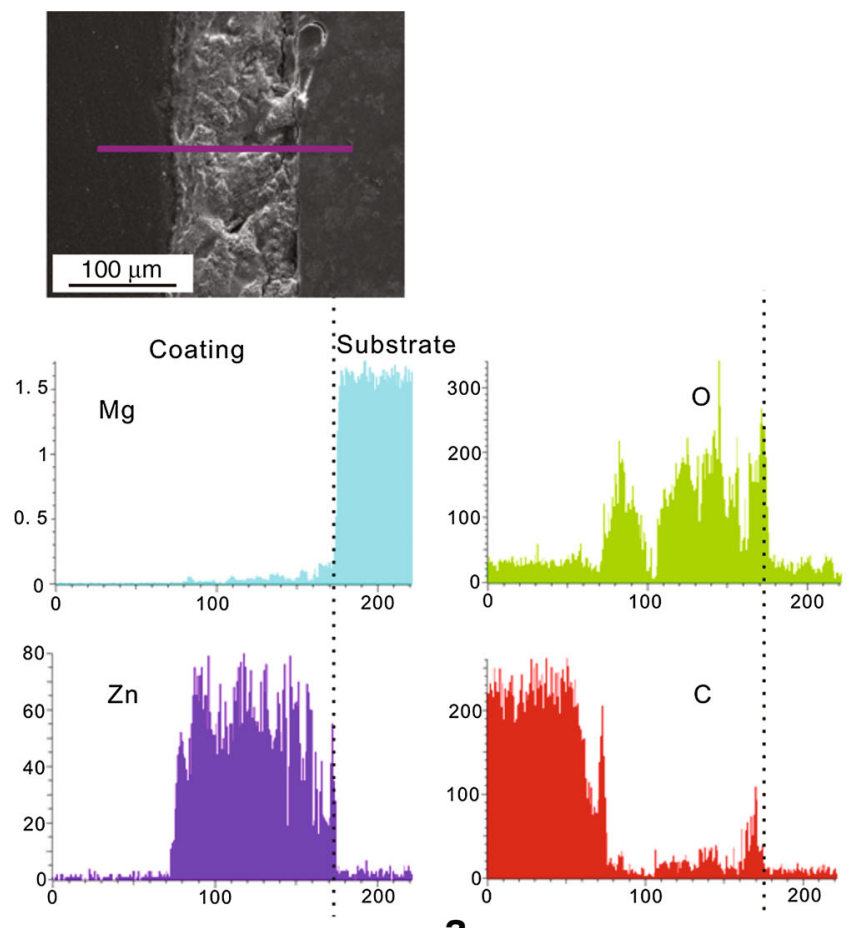

a
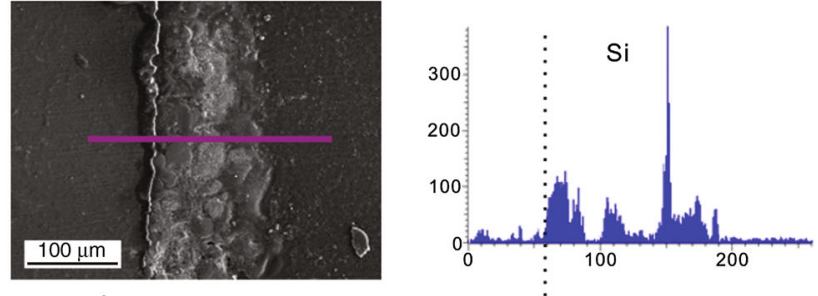

Substrate: Coating
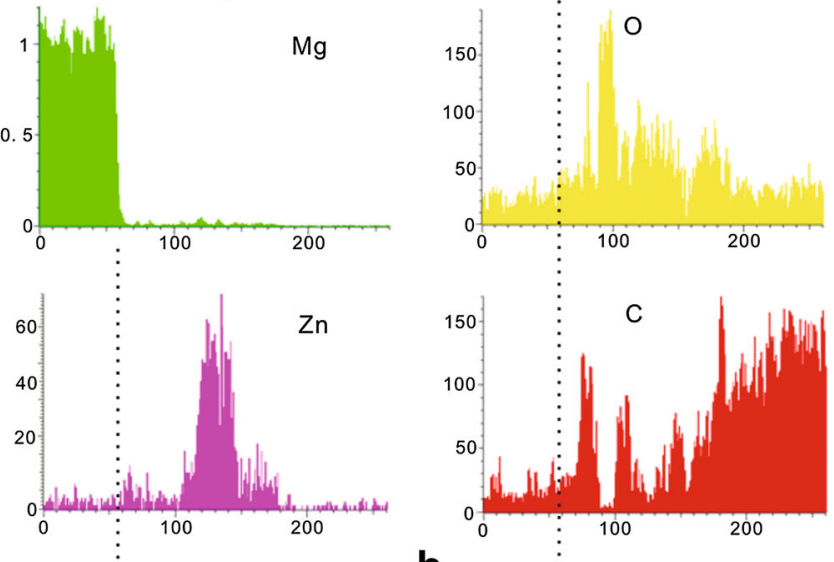

b

Fig. 3 SEM images of the cross-sectional view and the corresponding EDS line scanning analysis for the HZC film a and FAS/HZC film b
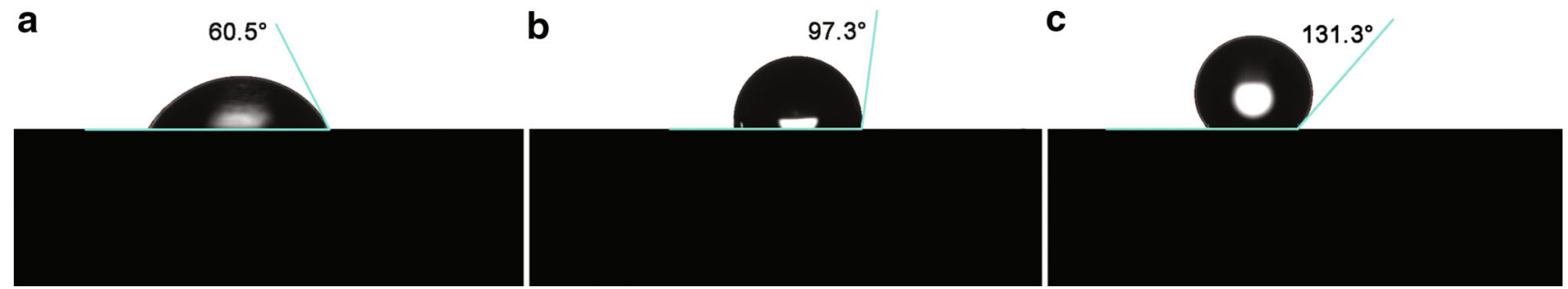

Fig. 4 Profiles of water droplets on the AZ31 substrates a, the HZC film $\mathbf{b}$ and the FAS/HZC film $\mathbf{c}$

known that the urea hydrolysis at an optimum temperature of approximately $85^{\circ} \mathrm{C}$ [42] led to the formation of carbonate and hydroxyl [43]. Then, $\mathrm{Zn}^{2+}$ ions underwent a coprecipitation with the released carbonate and hydroxyl and finally led to the formation of the HZC film. The chemical deposition occurred via the following processes [44]:

$$
\begin{aligned}
& \mathrm{CO}\left(\mathrm{NH}_{2}\right)_{2}+3 \mathrm{H}_{2} \mathrm{O} \rightarrow 2 \mathrm{NH}_{3} \cdot \mathrm{H}_{2} \mathrm{O}+\mathrm{CO}_{2}, \\
& \mathrm{NH}_{3} \cdot \mathrm{H}_{2} \mathrm{O} \rightarrow \mathrm{NH}_{4}^{+}+\mathrm{OH}^{-}, \\
& 2 \mathrm{NH}_{3} \cdot \mathrm{H}_{2} \mathrm{O}+\mathrm{CO}_{2} \rightarrow 2 \mathrm{NH}_{4}^{+}+\mathrm{CO}_{3}^{2-}+\mathrm{H}_{2} \mathrm{O}, \\
& \mathrm{Zn}^{2+}+2 \mathrm{OH}^{-} \rightarrow \mathrm{Zn}(\mathrm{OH})_{2}, \\
& 5 \mathrm{Zn}^{2+}+2 \mathrm{CO}_{3}^{2-}+6 \mathrm{OH}^{-} \rightarrow \mathrm{Zn}_{5}\left(\mathrm{CO}_{3}\right)_{2}(\mathrm{OH})_{6} .
\end{aligned}
$$

In addition, FT-IR spectra (Fig. 6) were also employed to characterize the chemical bonding of the hydrophobic

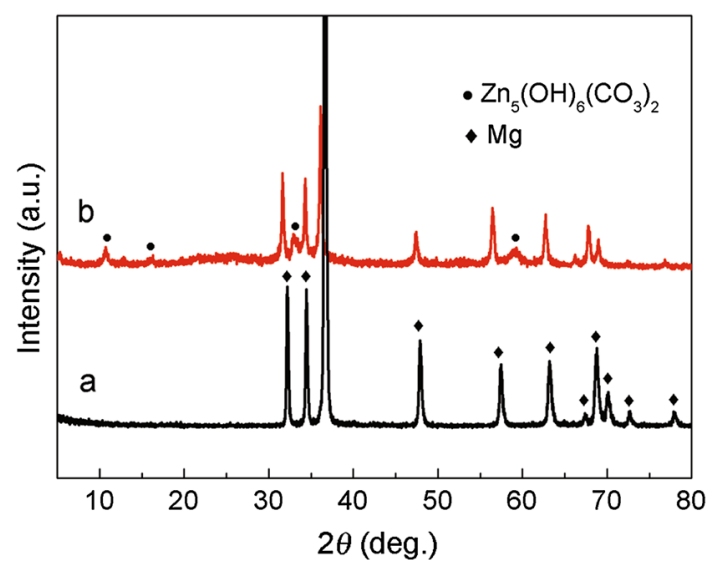

Fig. 5 XRD patterns of the AZ31 substrate $a$ and HZC powder scraped from the deposited film $b$ 
film. The band at $3,440 \mathrm{~cm}^{-1}$ corresponds to the $\mathrm{O}-\mathrm{H}$ vibration of the hydrogen bond [44], and the band at $1,616 \mathrm{~cm}^{-1}$ is attributed to the $\mathrm{H}-\mathrm{O}-\mathrm{H}$ bending mode of water molecule [45]. The bands assigned to carbonate groups can also be observed at the bands of $1,556-1,358 \mathrm{~cm}^{-1}$, assigned to the asymmetric stretching $v_{3}$ modes, the bands at $1,045 \mathrm{~cm}^{-1}$ corresponding to the symmetric stretching $v_{1}$ mode and at $834 \mathrm{~cm}^{-1}$ the out-ofplane deformation $v_{2}$ mode $[44,45]$. The band at $941 \mathrm{~cm}^{-1}$ is assigned to the $\mathrm{Zn}-\mathrm{OH}$ bond [45]. These results are pronounced consistent with the EDS and XRD results. The existence of the HZC film on the AZ31 substrate was also confirmed. The bands at 2,952 and $2,890 \mathrm{~cm}^{-1}$ are assigned to the $\mathrm{C}-\mathrm{H}$ stretching in $-\mathrm{CH}_{2}-$ groups [25]. The bands around $1,331,1,239,1,170$ and $1,117 \mathrm{~cm}^{-1}$ are designated to the $\mathrm{C}-\mathrm{F}$ stretching vibration of the $-\mathrm{CF}_{2}-$ and $-\mathrm{CF}_{3}-$ groups [46, 47]. The band around $1,012 \mathrm{~cm}^{-1}$ is assigned to asymmetric stretching vibration of the $\mathrm{Si}-\mathrm{O}-\mathrm{Si}$ bonds [25, 48], confirming that the FAS layer was chemically absorbed on the HZC film and the AZ31 alloy surface.

\subsection{Corrosion Resistance}

The values of open circuit potential, $E_{\text {corr }}$, and corrosion current density, $i_{\text {corr }}$, derived from the polarization curves (Fig. 7), are given in Table 1 . The $E_{\text {corr }}$ value of the AZ31 substrate is $-1,540 \mathrm{mV} / \mathrm{SCE}$. Compared to the bare substrate, the $E_{\text {corr }}$ values of the HZC and the FAS/HZC films were positively shifted 279 and $990 \mathrm{mV} / \mathrm{SCE}$, respectively. It is also noted that the $i_{\text {corr }}$ of the hydrophobic FAS/HZC film was markedly reduced by three orders of magnitude than that of the substrate. This result is ascribed to the nanostructured HZC film and the FAS film with a low surface energy. And the hydrolysis and condensation of FAS can form an effective $\mathrm{Si}-\mathrm{O}-\mathrm{Si}$ physical barrier on the

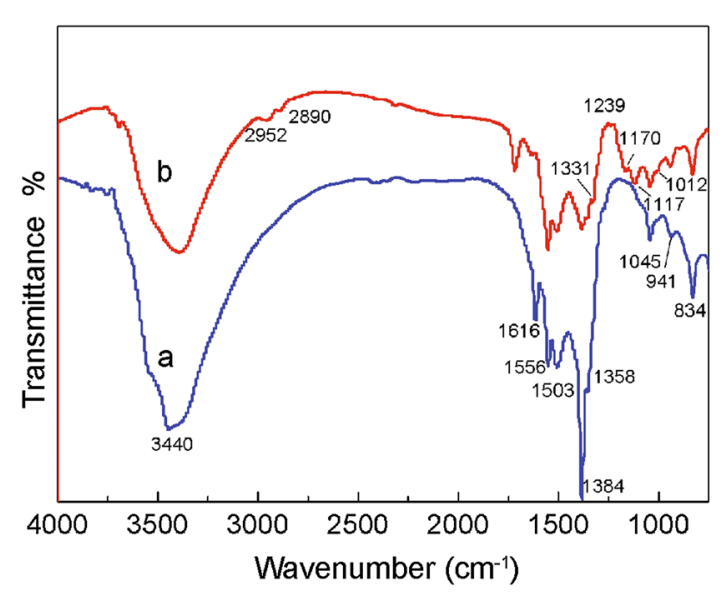

Fig. 6 FT-IR spectra of the HZC film $a$ and FAS/HZC film $b$

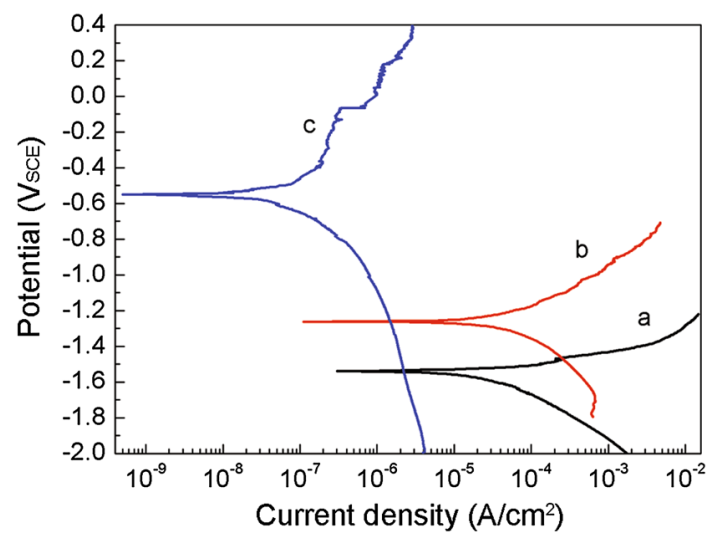

Fig. 7 Polarization curves of the AZ31 alloy $a$, the HZC film $b$ and the FAS/HZC film $c$ in $3.5 \mathrm{wt} \% \mathrm{NaCl}$ solution

Table 1 Electrochemical parameters of the polarization curves

\begin{tabular}{lcc}
\hline Sample & $E_{\text {corr }}(\mathrm{mV}$ vs. SCE $)$ & $I_{\text {corr }}\left(\mathrm{A} / \mathrm{cm}^{2}\right)$ \\
\hline AZ31 substrate & $-1,540$ & $2.64 \times 10^{-5}$ \\
HZC film & $-1,261$ & $2.24 \times 10^{-5}$ \\
FAS/HZC film & -550 & $2.61 \times 10^{-8}$ \\
\hline
\end{tabular}

$\mathrm{Mg}$ alloy surface to protect the substrates. However, the existence of the HZC film does not bring out an evident increase in the $i_{\text {corr }}$, because the numerous hydrophilic hydroxyl groups and micro-cracks and voids of the HZC film make it easy for the aggressive medium to attack the substrates. The scenario reveals that the hydrophobic FAS/ HZC film significantly improved the corrosion resistance of the AZ31 substrate, which is much more obvious than that of the super-hydrophobic film fabricated on the $\mathrm{Zn}$ plates [36].

\subsection{Corrosion Mechanism}

A schematic illustration of the interfaces between the hydrophobic surface and the solution is shown in Fig. 8. The hydrophobic surface of the FAS/HZC film is consisted of many 'hills,' and air can be trapped within the 'valleys' between these 'hills' [11, 19]. Hereby, the equation obtained by Cassie and Baxter [49] can be used to theoretically explain the hydrophobicity of the surface.

$\cos \theta_{r}=f_{s} \cos \theta_{s}-\left(1-f_{s}\right)$,

where $\theta_{r}$ is the CA on the rough surface, $\theta_{s}$ is the CA on a smooth surface with the same chemical nature, $f_{s}$ is the fraction of the solid-liquid contact area upon which the droplet sits, and $1-f_{s}$ is the air fraction below the droplets [50]. A contrast measurement of CA on a smooth surface was performed; the obtained value of $\theta_{s}$ is about $63.5^{\circ}$. 


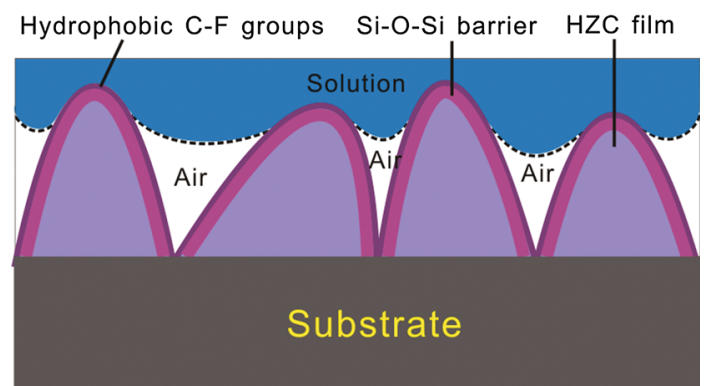

Fig. 8 Schematical illustration of the interface between the hydrophobic surface and the $3.5 \mathrm{wt} \% \mathrm{NaCl}$ solution

From Eq. (6), the estimated $f_{s}$ value is about 0.23 . Herein, the fraction of the air trapped within the interstices of the surface is approximately 0.77 . This large value increased the air/water interface, preventing the water droplet from infiltrating the space.

Another important reason for the improvement in corrosion resistance of the $\mathrm{Mg}$ alloy is attribute to the $\mathrm{Si}-\mathrm{O}-\mathrm{Si}$ bonds within the silane coating [51]. The cross-linked silane layer is dense and uniform, acting as a physical barrier to retard the solution penetration into the substrate.

The more hydrophobic the outer layer, the lower the penetration rate of water into the substrate [52]. Therefore, the aggressive $\mathrm{Cl}^{-}$can hardly reach the metal substrate due to the synergistic action of the hydrophobicity of the nanostructured porous HZC coating and the cross-linked $\mathrm{Si}-\mathrm{O}-\mathrm{Si}$ bonds within the FAS.

\section{Conclusions}

The hierarchical porous HZC film was successfully fabricated on the AZ31 Mg alloy surface through a chemicalbath deposition process. There were a considerable number of flower-like protrusions consisting of nanostructured plates on the surface, and the thickness of the HZC film was approximately $100 \mu \mathrm{m}$. The FAS-modified HZC film exhibited a hydrophobic property with a $\mathrm{CA}$ value of $131.3^{\circ}$. The results of electrochemical measurement demonstrated that the corrosion resistance of the AZ31 alloy was significantly improved by the hydrophobic FAS/HZC film, which was attributed to the synergy combination of the nanostructured porous HZC film and the low surface energy of the FAS film.

Acknowledgments This work was supported by the National Natural Science Foundation of China (No. 51241001), SDUST Research Fund (No. 2014TDJH104), Taishan Scholarship Project of Shandong Province (No. TS20110828), Scientific Research Foundation of Shandong University of Science and Technology for Recruited Talents (No. 2013RCJJ006), Scientific Research Foundation of Shandong for Outstanding Young Scientist (No. BS2013CL009) and Applied Basic Research Foundation of Qingdao (No. 13-1-4-188-jch) as well.

\section{References}

[1] L.Q. Chen, Y.T. Yao, Acta Metall. Sin. (Engl. Lett.) 27, 762 (2014)

[2] Y.J. Zhang, Y.W. Shao, T. Zhang, G.Z. Meng, F.H. Wang, Corros. Sci. 53, 3747 (2011)

[3] Z.W. Wang, Q. Li, Z.X. She, F.N. Chen, L.Q. Li, X.X. Zhang, P. Zhang, Appl. Surf. Sci. 271, 182 (2013)

[4] M.A. Chen, N. Cheng, Y.C. Ou, J.M. Li, Surf. Coat. Technol. 232, 726 (2013)

[5] Z.Y. Cui, X.G. Li, K. Xiao, C.F. Dong, Corros. Sci. 76, 243 (2013)

[6] Y.L. Gao, D.S. Xiong, C.S. Wang, Y.Z. Chen, Acta Metall. Sin. (Engl. Lett.) 22, 167 (2009)

[7] Z.Y. Zhao, R.G. Guan, X. Wang, C.M. Liu, Acta Metall. Sin. (Engl. Lett.) 26, 447 (2013)

[8] Y.W. Song, D.Y. Shan, R.S. Chen, E.H. Han, Corros. Sci. 51, 1087 (2009)

[9] P. Liu, X. Pan, W.H. Yang, K.Y. Cai, Y.S. Chen, Mater. Lett. 75, 118 (2012)

[10] T.T. Wan, Z.X. Liu, M.Z. Bu, P.C. Wang, Corros. Sci. 66, 33 (2013)

[11] T. Ishizaki, J. Hieda, N. Saito, N. Saito, O. Takai, Electrochim. Acta 55, 7094 (2010)

[12] R. Gao, J. Wang, X.F. Zhang, H.J. Yan, W.L. Yang, Q. Liu, M.L. Zhang, L.H. Liu, K. Takahashi, Coll. Surf. A 436, 906 (2013)

[13] B. Yin, L. Fang, J. Hu, A.Q. Tang, W.H. Wei, J. He, Appl. Surf. Sci. 257, 1666 (2010)

[14] S. Wang, L. Feng, L. Jiang, Adv. Mater. 18, 767 (2006)

[15] Z.X. She, Q. Li, Z.W. Wang, L.Q. Li, F.N. Chen, J.C. Zhou, ACS Appl. Mater. Inter. 4, 4348 (2012)

[16] T. Isimjan, T. Wang, S. Rohani, Chem. Eng. J. 210, 182 (2012)

[17] R. Qiu, D. Zhang, P. Wang, Corros. Sci. 66, 350 (2013)

[18] W.J. Xu, J.L. Song, J. Sun, Y. Lu, Z.Y. Yu, ACS Appl. Mater. Int. 3, 4404 (2011)

[19] T. Liu, Y.S. Yin, S.G. Chen, X.T. Chang, S. Cheng, Electrochim. Acta 52, 3709 (2007)

[20] F. Guo, X.J. Su, G.L. Hou, P. Li, Coll. Surf. A 401, 61 (2012)

[21] K. Dopierala, H. Maciejewski, J. Karasiewicz, K. Prochaska, Appl. Surf. Sci. 283, 453 (2013)

[22] X.Y. Fu, X.H. He, Appl. Surf. Sci. 255, 1776 (2008)

[23] M.S. Islam, N. Akter, M.R. Karim, Coll. Surf. A 362, 117 (2010)

[24] J.H. Yim, V. Rodriguez-Santiago, A.A. Williams, T. Gougousi, D.D. Pappas, J.K. Hirvonen, Surf. Coat. Technol. 234, 21 (2013)

[25] R.V. Lakshmi, Bharathibai J. J. Coll. Interface Sci. 339, 454 (2009)

[26] Z.L. Wang, R.C. Zeng, Trans. Nonferrous Met. Soc. China 20, s665 (2010)

[27] S.H. Yin, D.X. Wu, J. Yang, S.M. Lei, T.C. Kuang, B. Zhu, Appl. Surf. Sci. 257, 8481 (2011)

[28] R.C. Zeng, L.J. Liu, S.Q. Li, Y.H. Zou, F. Zhang, Y.N. Yang, H.Z. Cui, E.H. Han, Acta Metall. Sin. (Engl. Lett.) 26, 681 (2013)

[29] T. Ishizaki, N. Saito, Langmuir 26, 9749 (2010)

[30] Y. Liu, L. Li, G.L. Lu, S.R. Yu, Sci. China Technol. Sci. 53, $2972(2010)$

[31] Z.X. Kang, X.M. Lai, J. Sang, Y.Y. Li, Thin Solid Films 520, $800(2011)$

[32] R.C. Zeng, Z.G. Liu, F. Zhang, S.Q. Li, H.Z. Cui, E.H. Han, J. Mater. Chem. A 2, 13049 (2014) 
[33] R.C. Zeng, X.X. Sun, Y.W. Song, F. Zhang, S.Q. Li, H.Z. Cui, E.H Han. Trans. Nonferrous Met. Soc. China 23, 3293 (2013)

[34] R.C. Zeng, F. Zhang, Z.D. Lan, H.Z. Cui, E.H. Han, Corros. Sci. 88, 452 (2014)

[35] R.C. Zeng, Z.D. Lan, L.H. Kong, Y.D. Huang, H.Z. Cui, Surf. Coat. Technol. 205, 3347 (2011)

[36] J. Liang, Y.C. Hu, Y.Q. Wu, H. Chen, J. Nanomater. 2013, 1 (2013)

[37] I. Bernagozzi, C. Antonini, F. Villa, M. Marengo, Coll. Surf. A 441, 919 (2014)

[38] F.X. Zhang, J. Chan, H.Y. Low, Appl. Surf. Sci. 254, 2975 (2008)

[39] B. Su, M. Li, Z.Y. Shi, Q.H. Lu, Langmuir 25, 3640 (2009)

[40] M. Nosonovsky, B. Bhushan, Curr. Opin. Colloid Interface Sci. 14, 270 (2009)

[41] N. Kanari, D. Mishra, I. Gaballah, B. Dupré, Thermochim. Acta 410, 93 (2004)

[42] M. Bitenc, M. Mariňsek, Z. Crnjak, Orel. J. Eur. Ceram. Soc. 28, 2915 (2008)
[43] L.P. Xu, Y.S. Ding, C.H. Chen, L.L. Zhao, C. Rimkus, R. Joesten, S.L. Suib, Chem. Mater. 20, 308 (2008)

[44] X.F. Zhou, Z.L. Hu, Y.Q. Fan, S. Chen, W.P. Ding, N.P. Xu, J. Phys. Chem. C 112, 11722 (2008)

[45] S. Musić, S. Popović, M. Maljković, D. Dragčević, J. Alloys Compd. 347, 324 (2002)

[46] N. Saleema, D.K. Sarkar, D. Gallant, R.W. Paynter, X. Chen, ACS Appl. Mater. Int. 3, 4775 (2011)

[47] J.L. Song, W.J. Xu, X. Liu, Z.F. Wei, Y. Lu, Mater. Lett. 87, 43 (2012)

[48] Y. Lu, W.J. Xu, J.L. Song, X. Liu, Y.J. Xing, J. Sun, Appl. Surf. Sci. 263, 297 (2012)

[49] A. Cassie, S. Baxter, Trans. Fara. Soc. 40, 546 (1944)

[50] J. Jia, J.F. Fan, B.S. Xu, H.B. Dong, J. Alloy, J. Alloys Compd. 554, 142 (2013)

[51] X. Liu, Z.L. Yue, T. Romeo, J. Weber, T. Scheuermann, S. Moulton, G. Wallace, Acta Biomater. 9, 8671 (2013)

[52] J. Song, W.J. Van Ooij, J. Adhes. Sci. Technol. 17, 2191 (2003) 